\title{
Análisis de la Correlación entre las Emisiones Gaseosas y el Desempeño Energético de Fuentes Fijas de Combustión en Ecuador
}

\author{
Cabrera, Marcelo ${ }^{1, *}$ iD ; Montenegro, Lucía ${ }^{2}$ iD ; Guanulema, Jorge ; $^{\text {iD }}$ \\ ${ }^{1}$ Universidad Internacional del Ecuador, Escuela de Gestión Ambiental, Quito, Ecuador \\ ${ }^{2}$ Escuela Politécnica Nacional, Facultad de Ingeniería Civil y Ambiental, Quito, Ecuador \\ ${ }^{3}$ Escuela Politécnica Nacional, Facultad de Ingeniería Química y Agroindustria, Quito, Ecuador
}

\begin{abstract}
Resumen: En esta investigación, se analizó la eficiencia de combustión de veinte hornos a gas y fuel oil de refinerías alrededor del Ecuador, y treinta calderos de diferentes industrias en la ciudad de Quito, así como las emisiones de gases generadas y la rentabilidad de la inserción de precalentadores de aire para mejorar los procesos de combustión. El estudio se realizó en un período de 6 meses, obteniéndose los factores de emisión en los hornos a gas de 4,5 kg de $\mathrm{SO}_{2} / \mathrm{kg}$ de gas quemado; 22,4 de $\mathrm{NO}_{\mathrm{X}} / \mathrm{kg}$ de gas quemado y 44,5 $\mathrm{kg}$ de $\mathrm{CO} / \mathrm{kg}$ de gas quemado; mientras que los factores de emisión hallados para los hornos a fuel oil fueron de $93,9 \mathrm{~kg} \mathrm{de} \mathrm{SO}_{2} / \mathrm{kg}$ de fuel oil; $24,9 \mathrm{~kg}$ de $\mathrm{NO}_{\mathrm{X}} / \mathrm{kg}$ de fuel oil y 2,5 kg de $\mathrm{CO} / \mathrm{kg}$ de fuel oil. Por otra parte, los factores típicos de emisión de los calderos fueron de $1,7 \mathrm{~kg}$ de $\mathrm{SO}_{2} / \mathrm{kg}$ de diésel quemado; $1,5 \mathrm{~kg}$ de $\mathrm{NO}_{X} / \mathrm{kg}$ de diésel quemado y $1,0 \mathrm{~kg}$ de $\mathrm{CO} / \mathrm{kg}$ de diésel quemado. Finalmente, la implementación de un precalentador de aire aumenta la eficiencia del caldero analizado (CD20) en un 4,1\%, lo cual implicó un valor actual neto (VAN) de USD 8325,77 y un TIR del $31 \%$, con lo cual se determinó la factibilidad y rentabilidad del proyecto.
\end{abstract}

Palabras clave: Calderos, Eficiencia, Factores de emisión, Hornos, Intercambiador de calor, Precalentador de Aire.

\section{Analysis of the Correlation Between Gaseous Emissions and Energy Performance of Fixed Combustion Sources in Ecuador}

\begin{abstract}
In this research, the combustion efficiency of twenty gas and fuel oil furnaces from refineries around Ecuador, and thirty boilers from different industries in the city of Quito were analyzed, as well as the gas emissions generated and the profitability of the insertion of air pre-heaters to improve the combustion processes. The study was conducted over a period of 6 months, obtaining the emission factors for the gas-fired furnaces of $4.5 \mathrm{~kg}$ of SO $2 / \mathrm{kg}$ of gas burned; 22.4 of $\mathrm{NO}_{\mathrm{X}} / \mathrm{kg}$ of gas burned and $44.5 \mathrm{~kg}$ of CO/ $\mathrm{kg}$ of gas burned; while the emission factors found for the fuel oil furnaces were $93.9 \mathrm{~kg}$ of SO $2 / \mathrm{kg}$ of fuel oil; $24.9 \mathrm{~kg}$ of NO$/ \mathrm{kg}$ of fuel oil and $2.5 \mathrm{~kg}$ of CO/ $\mathrm{kg}$ of fuel oil. On the other hand, typical emission factors for the boilers were $1.7 \mathrm{~kg} \mathrm{SO}_{2} / \mathrm{kg}$ of diesel burned; $1.5 \mathrm{~kg} \mathrm{NO} / \mathrm{kg}$ of diesel burned and $1.0 \mathrm{~kg} \mathrm{CO} / \mathrm{kg}$ of diesel burned. Finally, the implementation of the air preheater increases the efficiency of the CD20 boiler by 4.1\%, which implied a net present value (NPV) of USD 8325,77 and an IRR of $31 \%$, which determined the feasibility and profitability of the project.
\end{abstract}

Keywords: Boilers, Efficiency, Emission factors, Furnaces, Heat exchangers, Air preheater.

\section{INTRODUCCIÓN}

La contaminación de la atmósfera es el resultado de la alteración en la composición natural del aire. Estos contaminantes representan un gran riesgo para la salud pública. Las Directrices de la OMS establecen los contaminantes PM (partículas), ozono $\left(\mathrm{O}_{3}\right)$, dióxido de nitrógeno $\left(\mathrm{NO}_{2}\right)$ y dióxido de azufre $\left(\mathrm{SO}_{2}\right)$ como contaminantes de interés global. Uno de los indicadores que produce mayor preocupación es el PM2.5, cantidad de partículas en suspensión menores a 2,5 micras, que mide el nivel de contaminación urbana. Estos contaminantes afectan al ambiente y a las condiciones de vida de los seres vivos. Otros autores establecen contaminantes adicionales a los declarados por la OMS, como son: monóxido y dióxido de carbono (CO), $\left(\mathrm{CO}_{2}\right)$; y óxidos de nitrógeno (NOx) (Wark y Cecil, 2008, p. 22; OMS, 2018, p.1). 
Para poder calcular las emisiones de contaminantes y como una estimación en la construcción de inventarios de emisiones, se emplea una herramienta de gestión ambiental llamada factor de emisión, que sirve para identificar la procedencia de los contaminantes que degradan la calidad del aire en una zona definida, en un período determinado. Este valor representa la relación entre la cantidad de contaminantes emitidos al ambiente con una variable de actividad asociada, por ejemplo, el uso de combustible, o los kilovatios consumidos o giga Joules de energía generados. Hay dos tipos de factores de emisión, el primero se basa en los procesos que generan emisiones y utiliza los datos de muestreos realizados a diversas fuentes para su cálculo, y el segundo se basa en censos socioeconómicos. Los factores de emisión con base en procesos se utilizan para la determinación de emisiones en fuentes fijas, y normalmente se relacionan con otros datos como balances de masa o encuestas. Por otro lado, aquellos con base en censos se emplean para valoraciones de las fuentes fijas de área (EPA, 2014).

El desempeño energético está definido por ISO 50001 (2018) como una medida o cuantificación de la relación entre el uso de la energía, la eficiencia energética y el consumo de la misma.

Conforme a lo mencionado, esta investigación tiene como objetivo el proporcionar datos de emisión reales de calderos y hornos a nivel local, de modo que sean útiles en la aplicación de inventarios de emisiones, el diseño de equipos de control, y la determinación de una línea base para el levantamiento de información en cada GAD. Cabe mencionar que en el Ecuador no existe una base de datos oficial con factores de emisión propios, y generalmente se emplean referencias internacionales como los contenidos en la AP-42 de la Environmental Protection Agency (EPA) de los Estados Unidos (Torres y Urvina, 2008, p. 16).

En la industria capitalina, los equipos de combustión, considerados como fuentes fijas puntuales de emisión, que se encuentran con más frecuencia son los calderos y los hornos. Ambos equipos son responsables del mayor consumo de combustible, por lo cual es importante a nivel económico y ambiental el mejorar su eficiencia (CORPAIRE, 2014, pp. 911).

La energía térmica generada por los equipos de combustión y desechada al ambiente en forma de calor, es un área de oportunidad para la reutilización u optimización de recursos. La energía térmica emitida se puede emplear en procesos que involucren transferencia de calor, o bien, reducirlas al emplear equipos con ventajas tecnológicas, ecológicas y económicas que disminuyan la pérdida de calor (Ayala, 2005, p. 10).

Para definir la eficiencia en un caldero se relaciona la cantidad de calor absorbido por el fluido (generalmente agua) para transformarse en vapor, y el calor generado por el combustible que se disipa por el equipo. Cuando se calcula la eficiencia energética de un caldero, es importante cuantificar el combustible que produce calor al reaccionar con el oxígeno de la cámara de combustión, así como considerar los gases producto de la combustión y el caudal del fluido que circula por el caldero que humedece los tubos por los cuales se transportan los gases y humedece el exterior de la superficie de la cámara y que toma la energía resultante de la combustión para aumentar su temperatura (IDAE, 2017, p.21).

La eficiencia en un horno, por otra parte, se define como un valor expresado en porcentaje, de la cantidad de calor que se libera en la llama y es utilizado por el fluido o material de trabajo. Los valores de eficiencia generalmente oscilan entre el 70,0 \% y el 95,0 \%. Las ineficiencias ocurren cuando la energía en forma de calor se disipa a través de las paredes del equipo y cuando se generan gases cómo un producto de la combustión (Domingo, 2015, p. 6).

Esta investigación pretende aportar en dos ámbitos: el ambiental, al proporcionar datos reales de factores de emisión en hornos y calderos; y energético, al utilizar las curvas determinadas en este documento, para aumentar la eficiencia de los procesos y disminuir las emisiones (Torres y Urvina, 2008 p. 16).

\section{MARCO METODOLOGÍCO}

Se seleccionaron 50 fuentes fijas de combustión, de ellas, 20 hornos de refinerías del Ecuador y 30 calderos del sector industrial de la ciudad de Quito. Para seleccionarlas, se tomó el criterio de continuidad de la operación de los equipos, así como la facilidad de realización de las mediciones.

La cuantificación de emisiones de los gases $\mathrm{SO}_{2}, \mathrm{O}_{2}, \mathrm{CO}_{2}, \mathrm{NO}_{\mathrm{X}}$ y $\mathrm{CO}$ fue realizada con un equipo para analizar gases de marca Testo $350 \mathrm{M} / \mathrm{XL}$. Este analizador cuenta con sensores electroquímicos que permiten medir la concentración de los contaminantes mencionados, así también cuenta con una termocupla y un sensor que permiten medir la temperatura de la chimenea y la temperatura del ambiente (TESTO AG, 2007, p. 61).

El analizador fue debidamente calibrado y verificado por un laboratorio acreditado ante el Sistema de Acreditación Ecuatoriano (S.A.E.) para la realización de este tipo de mediciones. El rango para el cual se encuentra habilitado el equipo es: 10 a 2000 ppm para los parámetros de $\mathrm{CO}, \mathrm{SO}_{2}$ y $\mathrm{NO}_{\mathrm{X}}$.

Para calcular los factores de emisión, se realizó de manera general para cada contaminante " $j$ ” empleando la Ecuación 1 que se presenta a continuación:

$$
F e_{j}=\frac{1}{\mathrm{~B}} * \sum_{i=1}^{n} C_{j} * G_{i} * \rho_{j} *\left(\frac{1 \mathrm{~kg}}{1000 \mathrm{mg}}\right)
$$

Donde:

$\mathrm{Fe}_{j}$ : factor de emisión del contaminante expresado en $\mathrm{kg} / \mathrm{kg}$ de combustible

$\mathrm{C}_{j}$ : $\quad$ concentración del gas contaminante medido $\left(\mathrm{ml} / \mathrm{m}^{3}\right)$

$\mathrm{G}_{i}$ : $\quad$ flujo de gases en el ducto o chimenea $\left(\mathrm{m}^{3} / \mathrm{h}\right)$

$\rho_{j}$ : densidad del contaminante $(\mathrm{mg} / \mathrm{ml})$, a condiciones normales de temperatura y presión $20^{\circ} \mathrm{C}$ y $760 \mathrm{~mm}$ $\mathrm{Hg}$.

$B$ promedio del consumo de combustible $(\mathrm{kg} / \mathrm{h})$ 
Una vez determinados los factores de emisión y las eficiencias promediadas, se construyeron las curvas para determinar las relaciones entre factor de emisión de contaminante, combustible empleado y eficiencia. Para el caso de los calderos, se realizó una distinción adicional que consistía en la discriminación debida a la potencia, de tal manera que fueron separados los equipos de la siguiente manera:

- Baja: Potencia del caldero menor a 100 brake horsepower (BHP)

- Media: Potencia del caldero entre 100 y 500 brake horsepower (BHP)

- Alta: Potencia del caldero mayor a 500 brake horsepower (BHP)

Como parte de esta investigación, se elaboró un estudio técnico-económico de una medida que permita mejorar la eficiencia de calderos, esta medida consistió en la planificación, diseño e implementación de precalentadores de aire que emplean termosifones bimetálicos aletados, los cuales pueden llegar a incrementar la eficiencia de combustión hasta en un $5 \%$, y consecuentemente una reducción considerable en el consumo de combustible (Ayala, 2005, p. 103).

A través de una aplicación desarrollada en una hoja de cálculo se determinaron las dimensiones del precalentador mientras que, con una evaluación de costos, se determinó el valor economizado en combustible por la mejora en la eficiencia del caldero. Para eso fue necesario además del costo del combustible, la información del número de horas por año de uso del caldero.

Durante el diseño y con el fin de evitar problemas en el sistema, se tomó en cuenta que la caída de presión de los gases de chimenea no se incremente y que la temperatura de los gases no sea menor que aquella del punto de rocío (Sánchez et al., 2011, p. 66).

Ayala (2005) sugiere considerar los criterios presentados a continuación, los cuales brindan un excelente marco para simplificar el cálculo de las dimensiones de los intercambiadores de termosifones aletados, y son:

- El promedio de cada termosifón define las condiciones de operación para el intercambiador.

- El diseño del banco de tubos presenta únicamente arreglo en forma de triángulo.

- $4 \mathrm{~mm}$ es la distancia mínima de montaje entre termosifones

- Se espera que entre $7 \mathrm{~m} / \mathrm{s}$ y $11 \mathrm{~m} / \mathrm{s}$ sea la velocidad media en la zona de gases.

- $\quad 110{ }^{\circ} \mathrm{C}(383 \mathrm{~K})$ debe ser la temperatura en la zona de salida de los gases de combustión para evitar el punto de rocío.

- La cantidad de termosifones de la primera y la última línea deberá ser igual.

- Para conservar una velocidad media entre las zonas, la relación entre las alturas de la zona de aire $(x)$ y la zona del precalentador $(\mathrm{y})$ deberá ser $\mathrm{x}=0,4 \mathrm{y}$.

Tras un análisis de costos, se determinó que los componentes mínimos necesarios para el diseño de un intercambiador de calor son:
- banco de tubos de acero inoxidable

- base inferior y superior

- paredes laterales e intermedia

- un aislador

- acabado exterior,

Sobre lo expuesto, se cotizaron los elementos mínimos para obtener un presupuesto y sobre este se realizó el análisis económico descrito más adelante.

En la Tabla 1, se detallan los precios de los componentes necesarios para el ensamblaje de un precalentador de aire, entre ellos, se puede observar que los termosifones son el elemento de mayor inversión, puesto que son complejos y tienen una cantidad considerable de tubos.

Tabla 1. Principales componentes de un precalentador de aire

\begin{tabular}{cc}
\hline Unidad & $\begin{array}{c}\text { Costo unitario } \\
\text { (USD/elemento) }\end{array}$ \\
\hline $\begin{array}{c}\text { Termosifones (tubo interior, tubo } \\
\text { aletado exterior) }\end{array}$ & 208,26 \\
Base baja & 131,74 \\
Base alta & 276,26 \\
Lámina media & 115,61 \\
Paredes laterales & 68,44 \\
Cubierta anterior & 106,24 \\
Cubierta posterior & 106,24 \\
Sellador & 55,24 \\
\hline
\end{tabular}

Se evaluó el ahorro económico en combustible que potencialmente se obtendría al implementar el sistema de recuperación de calor a través de los intercambiadores basados en termosifones bimetálicos aletados. Empleando una hoja de cálculo, se implementó una matriz para evaluar la utilidad, relacionando los egresos e ingresos tomando en cuenta variables como: depreciación, costos de operación, instalación y mantenimiento del sistema.

Adicionalmente, se determinó la viabilidad de la inversión a través de los parámetros económicos VAN y TIR.

\section{RESULTADOS Y DISCUSIÓN}

Con base en la información obtenida en el monitoreo de emisiones realizado a los hornos y calderos seleccionados, se determinaron las medias $(\mu)$ y desviaciones estándar $(\sigma)$ de cada medida de emisión. A continuación, se representa en cada una de las gráficas la relación entre los factores de emisión y la eficiencia.

La relación entre la eficiencia y los factores de emisión de hornos fuel oil $\mathrm{N}^{\circ} 4$ se puede observar en la Figura 1, mientras que los resultados obtenidos de las emisiones en hornos a gas se presentan en la Figura 2. 


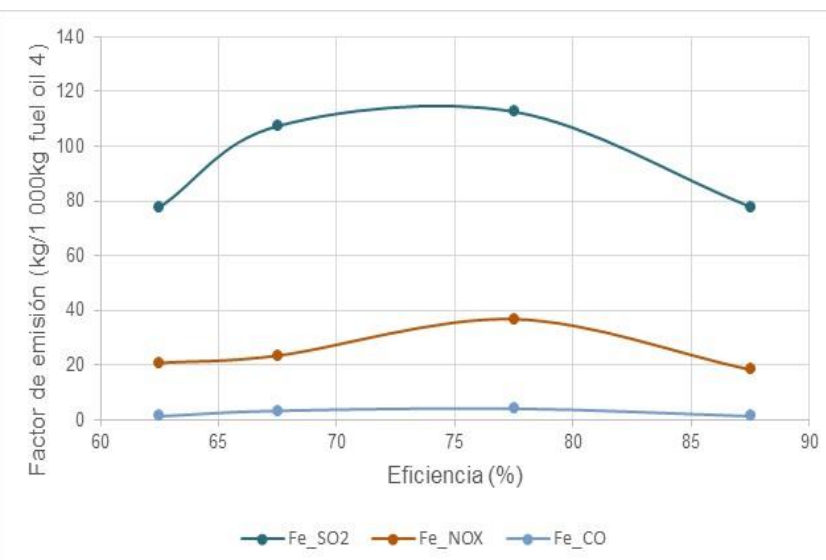

Figura 1. Relación entre el factor de emisión y la eficiencia (\%) para hornos a fuel oil No. 4

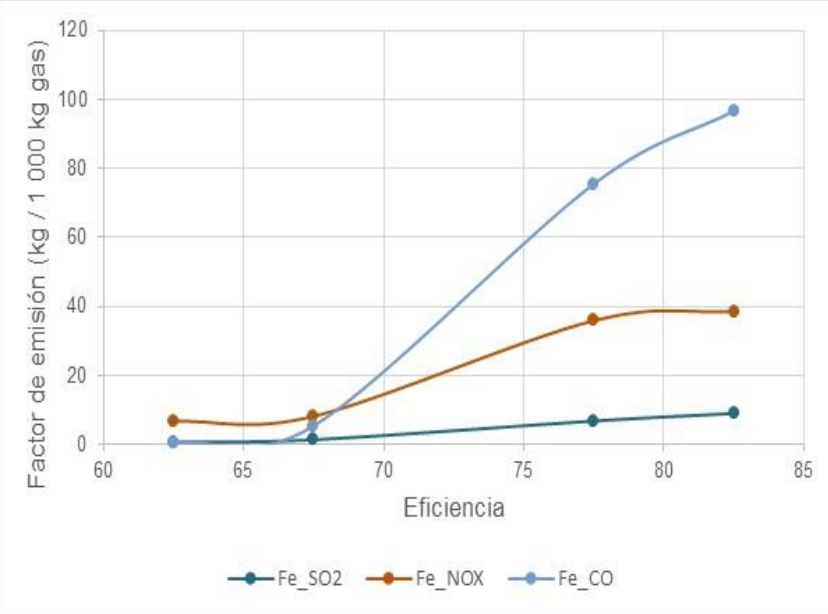

Figura 2. Relación entre el factor de emisión y la eficiencia (\%) para hornos a gas

Los valores obtenidos en la medición de la eficiencia de hornos del presente estudio se mantuvieron en el rango entre $60 \%$ y $90 \%$. El resultado mostrado en la Figura 1 responde a los hornos a fuel oíl No. 4 donde se observa que la eficiencia alcanza un pico máximo entre el $75 \%$ y $80 \%$, mientras que a los extremos $(62,5 \%$ y 87,5\%) los factores de emisión son mínimos para todos los gases.

En la Figura 2, se puede observar que a eficiencias superiores al $67 \%$, se incrementan considerablemente los factores de emisión, en especial del monóxido de carbono (CO). Los hornos a gas considerados en esta investigación trabajan a temperaturas por sobre $\operatorname{los} 300{ }^{\circ} \mathrm{C}$ o $573{ }^{\circ} \mathrm{K}$. Se evidencia que, a mayores temperaturas, se genera una mayor eficacia del sistema, y por ende una menor cantidad de oxígeno presente lo que facilita la formación de gases contaminantes. Adicionalmente, el incremento de la presencia de $\mathrm{SO}_{2}$ y $\mathrm{NO}_{x}$ se debe también a factores como el tiempo de permanencia de humos en la zona de combustión, el caudal de aire.

Las gráficas mostradas en la Figura 1 y en la Figura 2 permiten visualizar que las emisiones en un equipo varían entre los diferentes parámetros a analizar, presentando cada uno un comportamiento único, sin embargo, mantienen direcciones similares en crecientes a razón de los factores previamente mencionados.
Las Figuras 3, 4 y 5 corresponden a los factores de emisión de calderos que operan empleando diésel como combustible y de potencias media, baja y alta respectivamente.

La tendencia de los factores se mantiene en los calderos de potencia baja y media. Para el $\mathrm{SO}_{2}$ a eficiencias a partir del 90\% se presentó una disminución del factor de emisión, se atribuye este comportamiento al tiempo de mezcla aire-diésel que se da de mejor manera. En el caso del factor de emisión obtenido para los calderos de alta potencia prácticamente permanece constante alrededor de $1,5 \mathrm{~kg} / 1000 \mathrm{~kg}$ de diésel en tanto para $\mathrm{SO}_{2}$ como para $\mathrm{NO}_{\mathrm{X}}$, mientras que para el $\mathrm{CO}$ es cercano a $1 \mathrm{~kg} / 1000 \mathrm{~kg}$ de diésel.

Una alta potencia significa un mayor consumo de combustible, una mayor presión de inyección, y una mezcla de aire a la salida distinta a lo generado en calderos de baja potencia, lo cual explica su comportamiento.

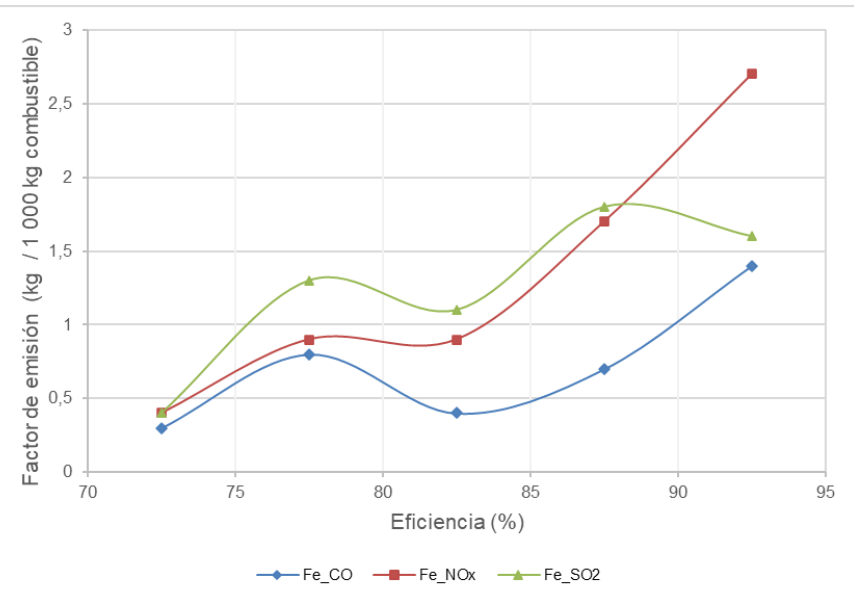

Figura 3. Relación entre los factores de emisión y la eficiencia de calderos a diésel de baja potencia

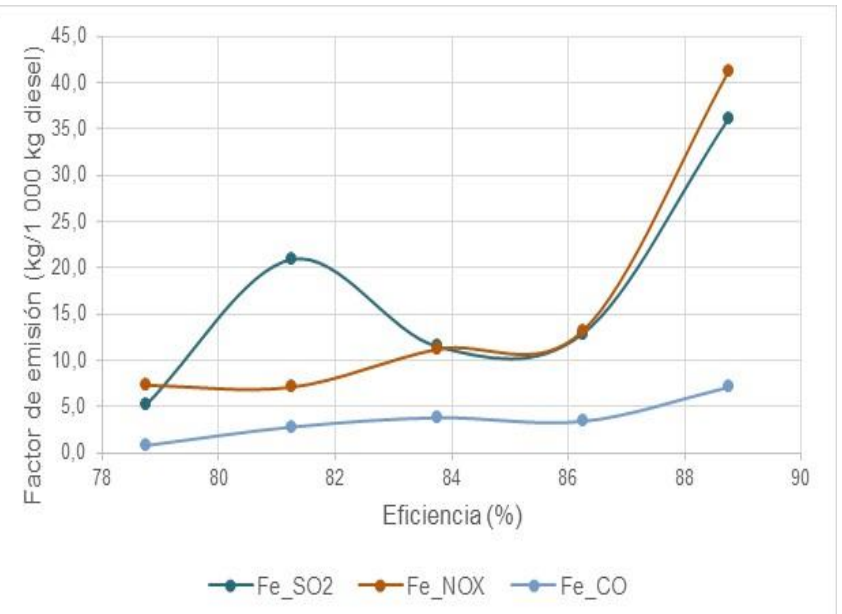

Figura 4. Relación entre los factores de emisión y la eficiencia de calderos de diésel de potencia media 


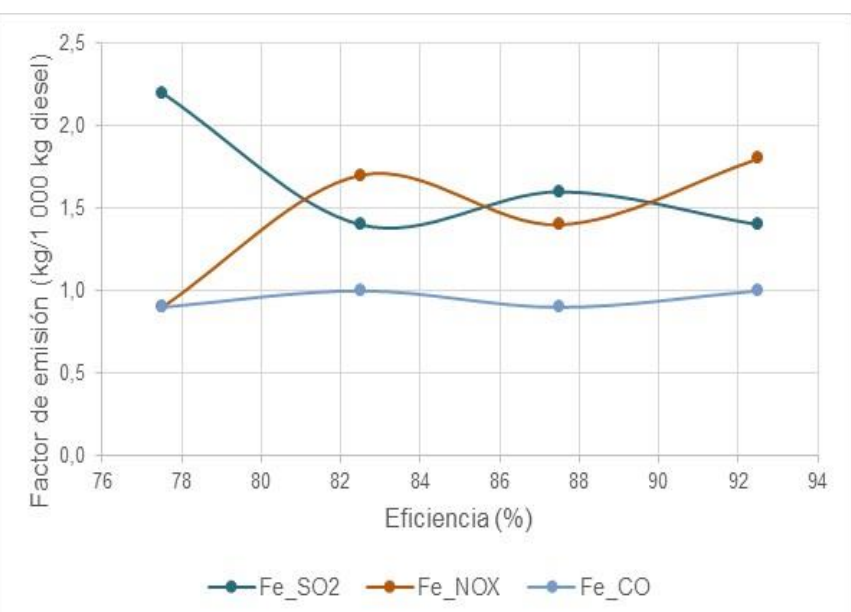

Figura 5. Relación entre los factores de emisión y la eficiencia para calderos de diésel de potencia alta

Las Figuras 6 y 7 corresponden a las relaciones entre factores de emisión y eficiencia obtenidos de la combustión del fuel oil No. 4 en calderos de baja y media potencia.

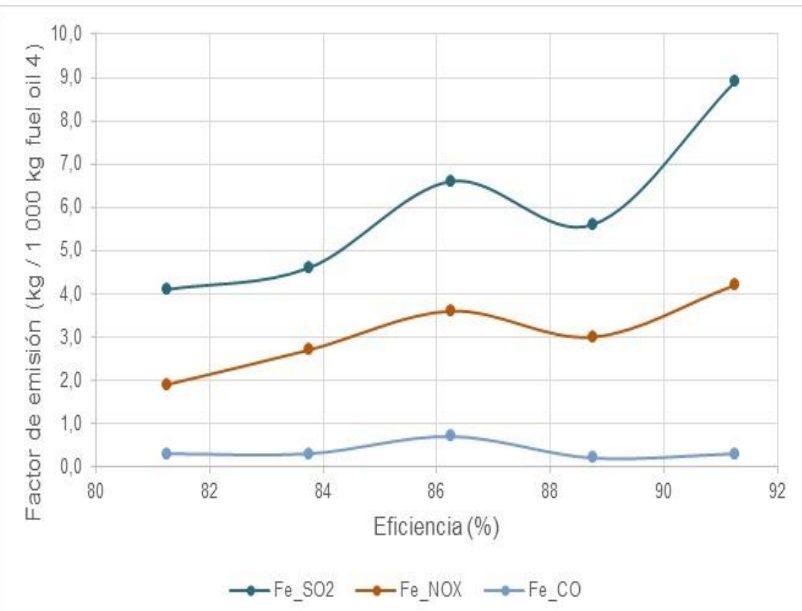

Figura 6. Relación entre factores de emisión y la eficiencia de calderos de fuel oil No.4 de baja potencia

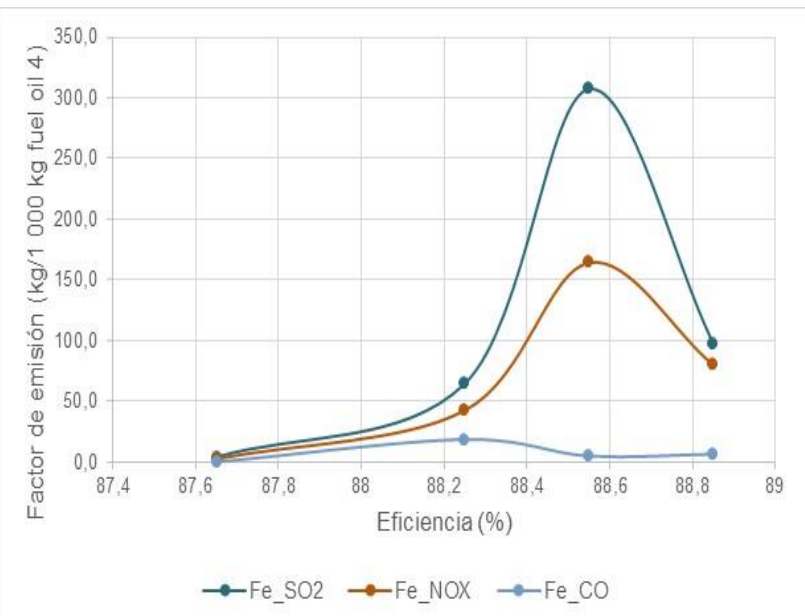

Figura 7. Relación entre los factores de emisión y la eficiencia de calderos fuel oil No.4 de potencia media

Los factores de emisión de $\mathrm{CO}$ se mantienen relativamente constantes en la Figura 6, mientras que los correspondientes al $\mathrm{SO}_{2}$ y $\mathrm{NO}_{\mathrm{X}}$ presentan comportamientos parabólicos con un máximo al $86 \%$ para el rango de eficiencia entre $84 \%$ y $88 \%$ pero crecientes a eficiencias superiores al $88 \%$.

Los resultados mostrados en la Figura 7 permiten apreciar que las curvas de $\mathrm{SO}_{2}$, así como de $\mathrm{NO}_{\mathrm{X}}$ mantienen tendencias muy semejantes. El CO en estos equipos presenta valores bajos, debido a esto su factor de emisión se comporta de igual forma, tal como se observa en la Figura 7.

En la Figura 8, se presentan los factores de emisión vs la eficiencia para calderos cuyo combustible es GLP y son de potencia media.

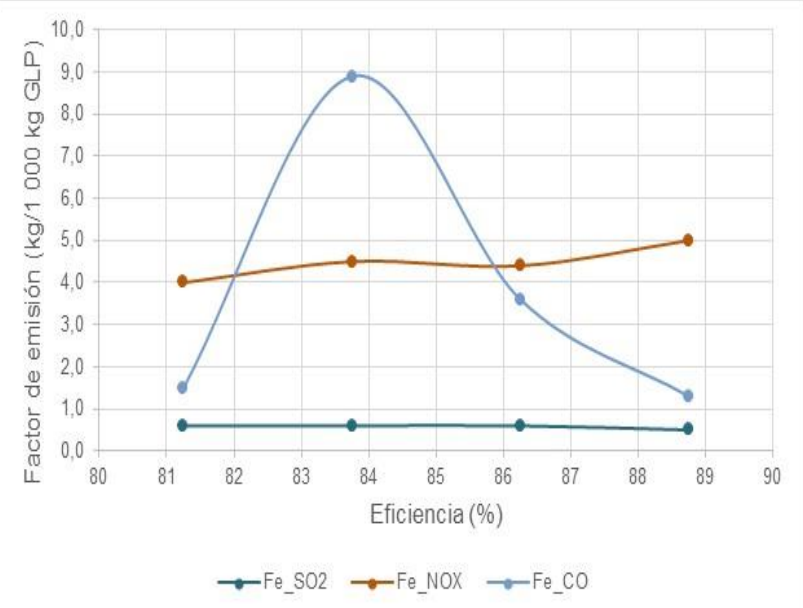

Figura 8. Relación entre los factores de emisión y la eficiencia para calderos GLP. de potencia media

A diferencia de los gráficos anteriores, en el caso de los factores de emisión de calderos a gas licuado de petróleo (GLP) presentado en la Figura 8, cada curva presenta una tendencia diferente. En el caso del $\mathrm{NO}_{\mathrm{X}}$ se mantiene alrededor de 4,5 kg/1 $000 \mathrm{~kg}$ de gas, mientras que para el CO se mantiene constante en aproximadamente $0,8 \mathrm{~kg} / 1000 \mathrm{~kg}$ de Gas.

De manera general, se concluye que la gráfica de los factores de emisión vs la eficiencia no presenta un comportamiento que pueda generalizase basado en regresiones matemáticas; sin embargo, el comportamiento entre contaminantes si puede ser asociado a una eficiencia. Para un caldero fuel oíl de potencia baja, a una eficiencia $91 \%$ determinada con el analizador de gases Testo $350 \mathrm{M} / \mathrm{XL}$, las emisiones de $\mathrm{SO}_{2}$ y $\mathrm{NO}_{\mathrm{X}}$ estarán en su punto máximo y si estos valores propician el incumplimiento respecto a la norma, se puede sugerir operar a una eficiencia del $89 \%$ y reducir significativamente las emisiones. Evidentemente, esta sugerencia debe enmarcarse en las implicaciones técnico-económicas propias de la empresa.

Finalmente, en la Tabla 2 se presenta un resumen de los datos obtenidos durante el desarrollo de esta investigación comparados con los factores de emisión US EPA. De lo cual se puede concluir que el factor de emisión más confiable es el diésel para calderos de baja, media y alta potencia, mientras que el de menor confianza es el factor de emisión de $\mathrm{SO}_{2}$ obtenido para hornos a gas. 
Tabla 2. Valores obtenidos de factores de emisión vs Factores de emisión referenciales AP-42 US EPA

\begin{tabular}{|c|c|c|c|c|c|c|c|}
\hline \multicolumn{2}{|c|}{$\begin{array}{l}\text { kg contaminante/ } \\
\text { kg combustible }\end{array}$} & \multicolumn{3}{|c|}{$\begin{array}{l}\text { Factores de emisión } \\
\text { calculados }\end{array}$} & \multicolumn{3}{|c|}{$\begin{array}{l}\text { Factores de emisión } \\
\text { referenciales EPA }\end{array}$} \\
\hline & & $\mathrm{SO}_{2}$ & $\mathbf{N O}_{\mathbf{x}}$ & $\mathrm{CO}$ & $\mathrm{SO}_{2}$ & NO $_{\mathbf{x}}$ & $\mathrm{CO}$ \\
\hline \multirow[t]{2}{*}{ Hornos } & Gas & 4,5 & 22,4 & 44,5 & 0,3 & 2,3 & 1,9 \\
\hline & Fuel oil & 93,9 & 24,9 & 2,5 & 23,6 & 6,8 & 0,6 \\
\hline \multirow{2}{*}{$\begin{array}{l}\text { Calderos } \\
\text { Baja }\end{array}$} & Fuel oil & 6,0 & 3,1 & 0,4 & 23,6 & 6,8 & 0,6 \\
\hline & Diesel & 1,2 & 1,3 & 0,7 & 4,2 & 2,8 & 0,7 \\
\hline \multirow{3}{*}{$\begin{array}{l}\text { Calderos } \\
\text { Media }\end{array}$} & Fuel oil & 118,4 & 72,3 & 7,5 & 23,6 & 6,8 & 0,6 \\
\hline & Diesel & 17,3 & 16,0 & 3,6 & 4,2 & 2,8 & 0,7 \\
\hline & GLP & 0,6 & 4,5 & 3,8 & 0,3 & 4,4 & 7,6 \\
\hline $\begin{array}{c}\text { Calderos } \\
\text { Alta }\end{array}$ & Diesel & 1,7 & 1,5 & 1,0 & 4,2 & 2,8 & 0,7 \\
\hline
\end{tabular}

Como parte de este trabajo, se elaboró una propuesta para el diseño de un precalentador de caldero, de lo cual se pueden observar los parámetros en la Tabla 3 y el resultado del análisis económico con las variables TIR y VAN en la Tabla 4.

Tabla 3. Definición de los parámetros para el diseño del precalentador de aire para el caldero CD20

\begin{tabular}{ccc}
\hline Parámetro & Cantidad & Unidad \\
\hline Diámetro interno del tubo (acero) & 0,020 & $\mathrm{~m}$ \\
Diámetro de contacto & 0,025 & $\mathrm{~m}$ \\
Diámetro externo del tubo (aluminio) & 0,028 & $\mathrm{~m}$ \\
Grosor de aleta & 0,0005 & $\mathrm{~m}$ \\
Paso entre aletas & 0,003 & $\mathrm{~m}$ \\
Diámetro externo de las aletas & 0,055 & $\mathrm{~m}$ \\
Longitud de la zona de gases & 0,9 & $\mathrm{~m}$ \\
Longitud de la zona de aire & 0,6 & $\mathrm{~m}$ \\
Cantidad de termosifones en primera & 5 & - \\
fila & 7 & - \\
Número de filas & 38 & - \\
Número total de termosifones &
\end{tabular}

El desarrollo y diseño de intercambiadores de calor basados en termosifones bimetálicos aletados involucró la caracterización del gas de chimenea de los calderos CD12 y CD20, ambos de potencia media y operación a diésel, que son los equipos más comunes en el Distrito Metropolitano de Quito. A continuación, se realizó la determinación de las dimensiones y variables del precalentador, cálculos de la transferencia de calor debida a la convección en gases de escape, así como en el aire. Luego se realizaron cálculos de condensación y ebullición en el agua confinada en cada termosifón, seguido de la determinación de la temperatura media logarítmica (LMTD) y finalmente el calor transferido por el precalentador. Para evaluar la satisfacción con las dimensiones del precalentador obtenido se comparó el flujo calórico determinado en un principio mediante balance energético $(16,3 \mathrm{~kW})$ con el calor obtenido mediante las variables de diseño $(18,1 \mathrm{~kW})$. El criterio de aceptación fue $3 \mathrm{~kW}$, con lo que se dio por viable el diseño de las dimensiones presentadas en la Tabla 3.

Tabla 4. Valor de los criterios financieros VAN y TIR para implementación del precalentador

\begin{tabular}{cc}
\hline Criterio Financiero & Cantidad \\
\hline Valor Actual Neto (VAN) & $\$ 8.325,77$ \\
Tasa interna de retorno (TIR) & $31 \%$ \\
\hline
\end{tabular}

En conclusión, el diseño de un intercambiador de calor que emplea termosifones aletados, cuya instalación, tentativa será realizada en el caldero $\mathrm{CD} 20$, es un proyecto económicamente factible de acuerdo con los indicadores económicos presentados en la Tabla 4, por lo cual se recomienda considerar esta alternativa para la mejora en la eficiencia de equipos de combustión fija que aplique.

\section{CONCLUSIONES}

Los hornos estudiados que operan a gas en el país cumplen con las concentraciones establecidas en la normativa aplicable vigente y sus valores promedios fueron respectivamente de 21 $\mathrm{mg} / \mathrm{m}^{3}$ vs $30 \mathrm{mg} / \mathrm{m}^{3}, 110 \mathrm{mg} / \mathrm{m}^{3}$ vs $400 \mathrm{mg} / \mathrm{m}^{3}$ y $173 \mathrm{mg} / \mathrm{m}^{3}$ vs N/A para los gases $\mathrm{SO}_{2}, \mathrm{NO}_{\mathrm{X}}$ y $\mathrm{CO}$, con una eficiencia del $70,80 \%$.

Las emisiones gaseosas producidas por los trece hornos de refinería a fuel oil fueron respectivamente: $0,964 \mathrm{~g} / \mathrm{m}^{3}, 0,283$ $\mathrm{g} / \mathrm{m}^{3}$ y $0,034 \mathrm{~g} / \mathrm{m}^{3}$ para $\mathrm{SO}_{2}, \mathrm{NO}_{\mathrm{X}}$ y $\mathrm{CO}$ con una eficiencia del $74,3 \%$, valores que cumplen con la exigencia de la entidad de control del país.

En los calderos a diésel, las emisiones de los calderos de los gases contaminantes $\mathrm{SO}_{2} \quad$ y $\quad \mathrm{NO}_{\mathrm{X}}$ incrementan proporcionalmente a la potencia del equipo; no obstante, no superan los límites establecidos por la normativa ambiental aplicable.

Cerca del 95,0 \% de los calderos analizados en este estudio, cumplen con la Resolución 002 vigente en el Distrito Metropolitano de Quito (agosto 2004) que trata de las emisiones gaseosas a la atmósfera.

Las siguientes eficiencias $62,5 \%, 65 \%$, y 62,5\% de los gases $\mathrm{SO}_{2}, \mathrm{NO}_{\mathrm{X}}$ y $\mathrm{CO}$, para los factores de emisión de hornos a gas de refinería son las que más se aproximan a los valores establecidos por la EPA. Esta diferencia evidencia la necesidad de determinar factores de emisión propios para nuestro país.

No está determinada una eficiencia para la cual los factores de emisión de los hornos a fuel oil, hallados en esta investigación, se acerquen a los señalados por la EPA. Esto se asocia a la concentración de azufre en el combustible y a las condiciones operativas de los equipos, pues se evidenció que al menos un $60 \%$ de los calderos se encuentran sobredimensionados por lo cual operan hasta 1 hora por día.

El factor de emisión de la EPA del monóxido de carbono (CO), y el determinado para los calderos a fuel oil de baja potencia se da a una eficiencia del $85,5 \%$, mientras que para el $\mathrm{SO}_{2} \mathrm{y}$ el $\mathrm{NO}_{\mathrm{X}}$ no existen valores de eficiencia que se aproximen al valor referencial correspondiente.

En calderos a diésel de baja potencia, la eficiencia al 92,5\% del factor de emisión de $\mathrm{NO}_{\mathrm{X}}$ es el porcentaje más aproximado a los valores establecidos por la EPA, mientras que para el factor de emisión del monóxido de carbono (CO) existen tres posibles porcentajes de aproximación hacia los factores de emisión EPA que son del $76 \%, 80 \%$ y 87,5 \%. 
Las relaciones obtenidas entre el combustible, potencia o tipo de fuente fija, se puede predecir un intervalo de eficiencia en el cual las emisiones de cada tipo de fuente de combustión tiendan a minimizarse.

La implementación de un precalentador de aire para el caldero CD20 es un proyecto viable, con un VAN fue de 8325,77 USD y un TIR del $31 \%$.

\section{REFERENCIAS}

Ayala, E. (2005). Desarrollo de precalentadores de aire compactos para recuperar energía de los gases de escape en calderos industriales de baja potencia. (Proyecto de titulación previa a la obtención del título de maestro en Ciencias en Ingeniería Mecánica). Instituto Politécnico Nacional, México D.F., México.

CORPAIRE (2014). Informe final. Inventario de emisiones contaminantes. Recuperado http://www.quitoambiente.gob.ec/ambiente/phocadownload/camb io_climatico/infor_final_ie_criterio_dmq2011_1.pdf (abril 2021).

Domingo, T. (2015). Diseño de equipos de instalación: Hornos y Calderos Recuperado de http://web.usal.es/ tonidm/DEI_08_comp.pdf (noviembre, 2019).

EPA (2014). Determination of Nitrogen Oxides, Carbon MoNOxide, and Oxygen Emissions from Natural Gas-Fired Engines, Boilers and Process Heaters Using Portable Analyzers (CTM-030). Recuperado de http://www.EPA.gov/ttnemc01 /ctm/ctm-030.pdf (octubre, 2019).

IDAE. (2017). Procedimiento de inspección periódica de eficiencia energética para calderos. Recuperado de http://www.idae.es/index.php/mod.documentos/mem.descarga?fil e=/documentos_10540_Procedimientos_inspeccion_calderos_GT 5_07_f5b208e3.pdf (octubre, 2019)

ISO 50001 (2018). Sistemas de Gestión de la energía. Recuperado de https://www.iso.org/obp/ui/\#iso:std:iso:50001:ed-2:v1:en (octubre, 2020).

Wark, K. y Cecil, W. (2008). Contaminación del aire. Origen y Control. México: Limusa.

OMS. (2018). Calidad del aire y salud. Recuperado de https://www.who.int/es/news-room/fact-sheets/detail/ambient(outdoor)-air-quality-and-health (abril, 2021).

Sánchez, F. et al. (2011). Diseño térmico y mecánico de un intercambiador de calor en base a tubos termosifones bifásicos. GetInfo, 12(3), 63-70. doi: $10.1006 /$ jezp.0716.8756.

TESTO AG. (2007). Análisis de gases de combustión en la industria: Guía práctica para medir emisiones y procesos. Recuperado de http://www.Testo.com/online/embedded

/Sites/ARG/SharedDocuments /Downloads/Guia_emisiones.pdf (octubre, 2019).

Torres, J., y Urvina V. (2008). Determinación de los factores reales de emisión de los motores ciclo Otto en la ciudad de Quito. (Proyecto de titulación previa a la obtención del título de Ingeniero Mecánico). Escuela Politécnica Nacional, Quito, Ecuador.

\section{BIOGRAFÍAS}

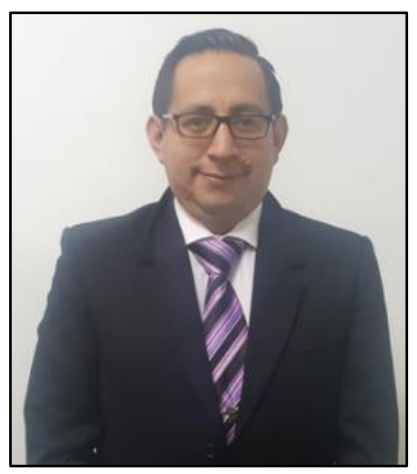

Marcelo F. Cabrera J. Nacido el 05 de marzo de 1986 en Quito-Ecuador, estudios completos de pregrado en la Escuela Politécnica Nacional, obteniendo el título de Ingeniero Químico Aprobado Cum Laude. Título de posgrado en la Universidad Internacional SEK como Magíster en Gestión Ambiental. Ha trabajado en Incinerox, Chemeng, Unidad Educativa Jean Jacques Rousseau, Escuela Politécnica Nacional y Universidad Internacional del Ecuador donde hasta la actualidad labora como docente en la Escuela de Gestión Ambiental en la UIDE y Coordinador del Laboratorio de Análisis Instrumental de la EPN. Ha realizado 5 publicaciones en revistas LatinIndex y 6 conferencias sobre contaminación ambiental y cursos de perfeccionamiento docente. macabreraja@uide.edu.ec / marcelofabian_cabrerajara@yahoo.com

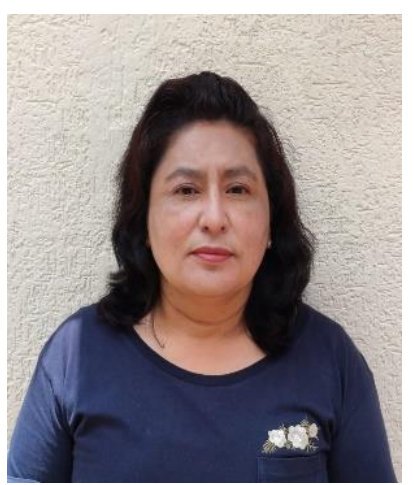

Lucía Margarita Montenegro Aguas. Ingeniera Química, Magister en Ingeniería Ambiental ha sido docente por 24 años en la EPN de las cátedras de Transferencia de Calor, Control de la Contaminación del Aire entre otras. Cuenta con la Certificación de Evaluador de la Norma ISO 17025 para laboratorios. En referencia a las publicaciones las ha realizado en la "Revista Politécnica" y en la "1st International Conference on Water and Sustainability" con temas relacionados al Tratamiento de aguas y Remediación de Suelos. Ha dictado más de 20 seminarios y conferencias de Monitoreo Ambiental; y dirigido más de 40 proyectos de titulación en temas de Transferencia de Calor, Refinación del Petróleo y Contaminación Ambiental. lucia.montenegro@epn.edu.ec 


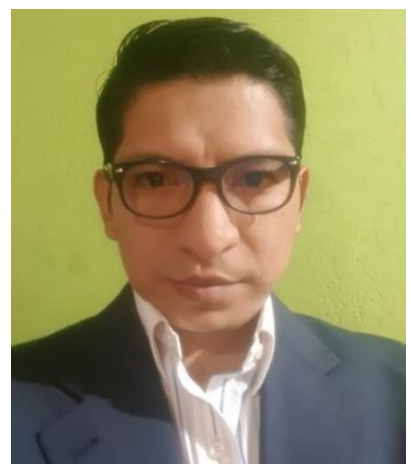

Jorge Guanulema. Ingeniero Químico graduado en la Escuela Politécnica Nacional Diplomado en Gerencia de Sistemas Integrados de Gestión HSEQ Universidad de Catalunya. Auditor Interno Sistemas integrados de Gestión HSEQ por SGS. Auditor interno ISO 9001:2015 por SGS. Analista Ambiental Senior en Grupo Consultor Chemeng (8 años). Técnico Ambiental en Secretaría de Ambiente del GAD del DMQ (actualmente). Experto Técnico ISO IEC 17025:2018 en el Servicio de Acreditación Ecuatoriano SAE para emisiones desde fuentes fijas de combustión, ruido ambiental laboral y Muestreo (actualmente) Evaluador de conformidad en Entrenamiento ISO IEC 17025:2018 Servicio de Acreditación Ecuatoriano SAE (actualmente) 